\title{
Speciation across the Earth driven by global cooling in orchidoid orchids
}

Jamie B. Thompson ${ }^{1}$, Harry O. Dodd ${ }^{1}$, Matthew A. Wills ${ }^{1}$, Nicholas K. Priest ${ }^{1}$

${ }^{1}$ The Milner Centre for Evolution, Department of Biology and Biochemistry, University of Bath, Bath BA2 7AY, United Kingdom

Corresponding authors: Jamie B. Thompson, Nicholas K. Priest

Email: jbt38@bath.ac.uk,np253@bath.ac.uk

Author Contributions: J.B.T., M.A.W. and N.K.P. designed the research. J.B.T. and H.O.D. performed the research, and J.B.T. and N.K.P. wrote the manuscript. All authors commented on the manuscript.

\begin{abstract}
Since Charles Darwin termed it his "abominable mystery", rapid speciation in angiosperms has eluded simple explanation. Climate has been implicated as a major catalyst of diversification, but its effects are thought to be inconsistent over time, between clades and across regions of the globe. Here we test the influence of climate change and geography-related climate factors on the diversification of the orchidoid orchids, a diverse subfamily of enduring fascination since Darwin's time. We show that global cooling spurred speciation in assemblages of orchidoids throughout the earth. Employing a phylogenetic framework, we demonstrate that speciation rate is correlated with historic changes in global temperature, but not atmospheric $\mathrm{CO}_{2}$ or sea-level. Cooling-driven diversification is consistent in all tribes, and in Disa, a large genus with well-supported signatures of adaptive evolution. We find no evidence for influences of geography-related climate factors, such as latitudinal and elevation gradients, or for "cradles" of diversity, in analyses of tip rates in the context of 1.5 million georeferenced records. Rather than being an abominable mystery, our results support a simple hypothesis that climate change can rapidly accelerate adaptive radiation in assemblages throughout the globe. Our findings raise the possibility that there are undiagnosed roles for climate change in other major radiations and indicate how contemporary climate change will influence long-term evolutionary processes.
\end{abstract}

\section{Significance Statement}

The staggering biodiversity of angiosperms has been difficult to reconcile with the gradual Darwinian process thought to create it. Changes in climate through the Earth's history could have instigated this diversification, but perceived variability across clades and geography has restrained generalization. In this paper, we reconstruct the evolutionary history of a rich orchid subfamily favoured by Darwin (Orchidoideae, 5,000 sps), and use 1.5 million georeferenced records to test when and where those orchid species arose. We find that global cooling between the Oligocene and present day spurred an avalanche of speciation in orchidoid assemblages across the Earth. This work resolves the orchidoid phylogeny and provides a clear example of widespread speciation driven by climate change. 


\section{Main Text}

\section{Introduction}

Charles Darwin's "abominable mystery" was why diversification can happen so rapidly $(1,2)$. The abrupt appearance of diverse clades of angiosperms was a challenge not only to Darwin's theory, but to evolution itself. William Carruthers, the Keeper of Botany at the British Museum, argued that it provided evidence for divine intervention in the tree of life (3). Under mounting pressure from Carruthers and other paleobotanists, Darwin asserted an admittedly 'wretchedly poor conjecture' that the patterns could be explained by an ancient origin of angiosperms on 'small isolated continent in the southern hemisphere' (letter to Joseph Hooker, 22 July 1879, Darwin Correspondence Project). Since then, there has been active inquiry into the age and ecoregion of the diversification of angiosperm clades and the "abominable mystery" itself $(3,4,5)$.

Diversification could be accelerated by climate change. Climatic events in the Earth's history are generally thought to have inconsistent influences in deep time evolution. Their influence on diversification can vary between closely related groups of taxa $(6,7)$, the type of climate change $(8)$ and the ecoregion in which it occurs $(9,10)$. Some studies find that climate change has little influence in the tropics, where we might anticipate its effects most (7). Moreover, in many of the classic cases of adaptive radiations, such as cichlid fish, Darwin's finches, and Anolis lizards, diversification occurred at such localised scales that it is difficult to determine the impact of global climate change on the evolutionary process. The critical unresolved question is the geographic scope of the influence of climate change on diversification in important angiosperm clades.

The orchidoideae (orchidoid orchids) are an ideal clade with which to test this question. Despite recent origins (65 mya), the orchidoids are globally distributed and extraordinarily diverse (11), both useful for testing consistency in the influence of climate change. They are central to Darwin's "abominable mystery" as his enigma originated with patterns he observed in orchids and related angiosperms (Letter to Hooker, 22 July 1879, Darwin Correspondence Project). Darwin was an expert on orchids. His first book published after "On the Origin of Species" included a focus on pollination of species including many orchidoids, and represented his first experimental demonstration of natural selection (12). Indeed, orchidoids possess the pollinia Darwin manipulated to prove their importance in plant-insect coevolution, and whose presence has been shown to accelerate speciation (13).

\section{Results}

\section{Phylogeny and Diversification}

Diversification rate is varied across the orchidoids (Figure 1). We reconstructed a maximum likelihood phylogeny of 1,450 taxa (29\% of the 5,000 known species), using nine nucleotide loci mined from Genbank. The phylogeny is well-supported, with $66 \%$ of internal nodes supported by $>70 \%$ bootstrap support (BS) and $44 \%$ by $>90 \%$ BS, and topology generally agrees with recent estimates (13). We time-calibrated this phylogeny with penalised likelihood (14), implementing 15 divergence constraints (Figure 1; Table 1) (13).

We estimated speciation dynamics using the reversible-jump Markov Chain Monte Carlo (MCMC) framework Bayesian Analysis of Macroevolutionary Mixtures (BAMM) (15), accounting for incomplete sampling by specifying the known richness of each genus (11). In all analyses with BAMM, we only considered speciation rate, not extinction rate, which avoids potentially unreliable parameter estimation $(16,17,18)$ and is predicted to accurately estimate speciation rate variability (19). We found 35-fold variation in the rate of speciation, with strong support for 24 rate shifts (Bayes Factor 20.8). In the shift configuration maximising a posteriori probability, the earliest rate shifts occur approximately at the Eocene-Oligocene boundary, but the majority fall between the Oligocene-Miocene boundary and the present day. These time points are relevant because they correspond with a protracted period of climatic cooling in Earth's history. 


\section{Cross-clade consistency in consequences of climatic cooling}

To formally test the hypothesis that climatic cooling drove orchidoid speciation, we correlated 9,000 realisations of the historical speciation curve with a reconstruction of Cenozoic $\delta^{18} \mathrm{O}(\mathrm{a}$ proxy for mean global temperature) (20). Specifically, we tested whether the distribution of correlation coefficients differed from zero (which would indicate no relationship) using a Wilcoxon test. Across the subfamily, speciation rate is strongly negatively correlated with Cenozoic $\delta^{18} \mathrm{O}$ (DCCA: $-0.50, p<0.0001$; Figure 2b). Using Transfer Entropy (21), an information theory metric that has been applied to paleontological datasets, $(8,22)$, we find that global cooling stimulates diversification through an unknown variable (Transfer Entropy $=-0.54$, outside the $95 \% \mathrm{Cl}$ of randomisation tests, see 23). To control for other climatic drivers, we tested for the influence of historic values of mean atmospheric $\mathrm{CO}_{2}$ and global sea level variation, both of which are correlated with mean global temperature (24) and have been shown to influence diversification of other clades (ex: $8,10,25,26)$. Though statistically significant, neither atmospheric $\mathrm{CO}_{2}$ nor sealevel were strongly correlated with diversification in the Orchidoideae $\left(\mathrm{CO}_{2}: 0.031, p<0.0001\right.$; sealevel: $-0.029, p<0.0001)$.

In contrast to other studies $(6,7)$, we find cross-clade consistency in the influence of climate change on diversification. Our DCCA analysis reveals negative correlations between the speciation curve and historical global temperature in each of the three major subclades (Cranichideae -0.48, $p<0.0001$; Diurideae $-0.43 p<0.0001$, Orchideae/Diseae $-0.44, p<0.0001$; Figure 2). We also performed a targeted analysis to test the influence of global cooling in taxa that exhibit signatures of adaptive evolution. As all orchidoids produce pollinia, masses of pollen that easily attach to insect pollinators, we cannot test for their role in accelerated speciation (as has been established in orchidaceae generally, see 13). However, diversification in the genus Disa is indicative of adaptive radiation because it exhibits a diversity of pollination syndromes with well-supported evidence of adaptive evolution $(27,28)$. Consistent with findings across the subfamily, we find that the speciation curve of Disa is negatively correlated with global mean temperature $(\mathrm{DCCA}=-0.46, \mathrm{p}<0.0001)$.

\section{Lack of climate-related geographic effects}

Recent studies show that diversification in angiosperms is generally faster where it is cooler, particularly outside the tropics $(7,29)$ and in highlands $(30,31,32)$. We conducted a series of tests to confirm whether the orchidoids are specifically sensitive to temporal, rather than spatial climatic variation. Global maps based on 1.5 million georeferenced occurrence records (35) suggest that there are regions of high speciation rate and richness (Figure 3a,b). To formally test for influences of spatial climatic variation, we used Structured Rate Permutations On Phylogenies (STRAPP) and Es-sim to assess the impact of temperature-associated regions on speciation (33 34). In contrast with previous work, where the high diversification rate of orchids is argued to result from non-tropical distributions (13), we find no significant difference in speciation rate between tropical and nontropical taxa when defining by latitude $(-23.5 /+23.5$ degrees, STRAPP $\mathrm{p}=0.48$ ). Nor do we find a significant difference when defining mean latitude continuously (absolute latitude Es-Sim $p=0.11$, STRAPP $p=0.48$; raw latitude Es-Sim $p=0.18$, STRAPP $\mathrm{p}=0.37$ ). There is also no significant difference in speciation rate defining the tropics by temperature, with contemporary data (year-round $>18^{\circ} \mathrm{C}$ average, $p=0.55$ ). Contrary to the notion that orchid diversification is promoted by highland distribution (32), we also find no significant correlations between speciation rate and measures of elevation (minimum: STRAPP $p=0.38$, EsSim $p=0.29$; maximum: STRAPP $p=0.3$, Es-Sim $p=0.08$ ). These findings indicate that the influence of global cooling on diversification in the orchidoid differs through time, not space.

\section{Worldwide speciation}

To confirm cross-Earth consistency in diversification, we sorted occurrence records from GBIF (35) by endemic species found in the seven major orchid bioregions, defined as Africa, Australia, Europe, North America, Neotropics, the Pacific, and Southeast Asia (see 36). We find little 
evidence for a speciation advantage conferred by any bioregion (STRAPP, Kruskal-Wallis, $p=0.085$ ). Between each of the major orchid bioregions we find the speciation rate curves are consistently strongly positively correlated across time (Figure 3c). This is particularly evident in comparisons between bioregions with similar numbers of endemic species (Figure 3d). In all bioregions except the Pacific, a species-poor sample, members are phylogenetically clustered (37), even though none constitute a monophyletic group (Africa $D=-0.51, n=221$; Australia $D=-$ $0.40, n=228$; Eurasia $D=-0.41, n=69$; North America $D=-0.51, n=29$; Neotropics $D=-0.57, n=109$; Pacific 0.28, $n=33$; Southeast Asia $D=-0.03, n=47$ ). Correlating speciation rate through time with historical temperature finds consistent negative DCCA correlations (Africa: -0.16 ; Australia: -0.57 ; Eurasia: -0.40; North America: -0.05; Neotropics: -0.34; Pacific: -0.42; and Southeast Asia: -0.48, with $p<0.0001$ for all bioregions).

\section{Diverse regional responses to climate change}

The consequences of climate change at local scales can be obscured in global analyses. Previous work shows that the effects on diversifications are determined by the type of climate change $(8)$ and the ecoregion $(9,10)$. DCCA analyses within each bioregion confirmed that the influence of atmospheric $\mathrm{CO}_{2}$ and sea-level variation on speciation rate varies at smaller geographic scales (Table 1). Despite previously finding only a weak clade-wise influence on diversification, we show that mean atmospheric $\mathrm{CO}_{2}$ is positively correlated with speciation rate in two bioregions (Pacific and Southeast Asia) and global sea level variation positively correlated with speciation rate in two different bioregions (Africa and North America; Table 1). Although the correlations were much stronger for global cooling, the findings show that different aspects of climate change can contribute at much more localised scales. Because data on historic climate change over geological timescales is a global average, rather than within each bioregion, similarities responses to climate change in different regions could result from regression to the mean. However, our finding of the bioregion-specific influences of atmospheric $\mathrm{CO}_{2}$ and sealevel variation suggest that this problem is minimal.

\section{Discussion}

Darwin was fascinated by angiosperms, especially orchids; but, he struggled to reconcile their rapid emergence with his theory of gradual adaptive evolution (1). There are conflicting responses to climate variation seen in broad taxonomic studies $(13,38)$. Temperature change has been long associated with biological diversification $(6,22)$, but there is a need to distinguish between climatic effects resulting from local geography and global climate change through history. We reconstructed a well-supported phylogeny of orchidoids, the first for this major clade. Using a phylogenetic approach, we find no influence of geographic variation on diversification. Instead, our study shows that global cooling stimulated contemporaneous accelerated radiations in orchidoid assemblages found across the globe.

This finding begs the question of whether there are undiagnosed roles for climate change in other major radiations. Recent work shows that a temporally complex mixture of tectonic activity, climate change, biotic resource flow and interspecific hybridization drove the famous diversification of cichlid fish $(39,40)$. Rapid evolution in response to climate change is wellestablished in particular species of Darwin's finches and Anolis lizards $(41,42)$. The persistence of ancient alleles in these groups $(43,44)$ is consistent with bursts of genetic variation occurring early in island colonisation; but, it is also consistent with a colonisation event followed by climate change-driven radiation. It is not possible to differentiate between these models of evolution without detailed records of historic climatic variation. This conclusion is confirmed by our finding of spatial variation in the effects of $\mathrm{CO}_{2}$ and sea level, which implies that the consequences of climate change can vary greatly by geography, even with global consistency in the consequences of climatic cooling.

\section{Mechanisms of evolution driven by climate change}

It is not clear how global cooling drives diversification. Milankovitch (orbital) cycles change the distribution of exposure to annual solar energy in predictable ways, but responses on Earth, 
including biotic changes, tectonic activity and climate change, can be varied (45). The consistency we found in the influence of global cooling rejects a unique role for tectonics, which is expected to have localised geographic impacts. However, our Transfer Entropy findings of an unknown driver in mediating the effect of global cooling on speciation rate also rejects a direct influence of temperature on diversification.

Ochidoid diversification could be caused by climate-driven niche availability. Though there are many possible ecological drivers, the most probable mechanism is the one Darwin identified: coevolution between orchids and their pollinators (12, see 46). The global expansion of C4 grasslands could explain more recent patterns, but not the inception of accelerating orchidoid speciation. During the Miocene and early Pliocene (peaking 4-8 Ma), new niches were created as a consequence of cooling-driven expansion and diversification of C4 grasslands $(22,47,48)$. Thus, grassland expansion is spatially and temporally consistent with the later, but not earlier, accelerations in orchidoid speciation.

Climatic cooling could also drive rapid speciation by increasing genetic variability. Several studies show that biotic and abiotic factors, particularly temperature, can alter meiotic recombination in patterns which can accelerate adaptation $(49,50)$. Major changes in genome size and GC content are associated with orchidoid diversification $(51,52)$. Recent work has highlighted a role for stress in generating diverse orchidoid lineages with distinct ecological optima (53). The orchidoids also show evidence of recent allotetraploidy (54), suggesting that new species formed during global cooling as a consequence of alterations in meiosis. Though our phylogenetic approach did not permit careful assessment of admixture or levels of polyploidy, it is important to note that recurrent allotetraploidy would be consistent with our finding of a well resolved orchidoid phylogeny with high speciation rates.

\section{Contemporary Implications}

Resolving whether climate change stimulates adaptive radiation through stronger selection or increased genetic variability has implications for our understanding of the conservation of nature. There has been substantial interest in whether plants have sufficient standing genetic variation to respond to predicted gradual global warming (55). But, the history of earth is marked by catastrophic shifts in climate (55). And, climate change can impose strong breeding systems selection $($ ex. 57, 58). The relevant question for conservation could be whether plant species have sufficient capacities to generate novel genetic variation in response to climate-induced stress. Our finding that global cooling stimulated the formation of the thousands of genetically distinct orchidoid species within a short period (30 Ma) implicates a predictable role for catastrophic changes in selection and genetic variability in speciation. How climate change stimulates rapid adaptation and sparks rapid speciation is an important avenue of future research.

The "abominable mystery" that haunted Darwin's career was that the burst of flowering plants occurred too rapidly to reconcile with his vision of adaptive radiation $(1,2)$. A substantial body of work now shows that Darwin's vision of coevolution as a slow and gradual process was too slow and not sufficiently saltational. Although it is difficult to disentangle the relative influences of climate and coevolution, our findings support a simple resolution: climatic cooling accelerates Darwinian evolution. Our hypothesis is particularly supported by our evidence of cooling-driven diversification within Disa, which exhibits clear signatures of adaptive responses to pollinator preferences (59) and where Darwin's coevolutionary hypothesis has previously failed (28). Furthermore, a special role for pollinia in cooling-sensitive speciation could explain why the innovation of pollinia in early orchidoids lagged behind its influence on speciation during global cooling (see 13). Our results are in conflict with Darwin's coevolution hypothesis, but offer a solution to his "abominable mystery", with support across major subtribes and bioregions.

\section{Materials and Methods}

\section{Supermatrix assembly}


We mined Genbank for orchidoid sequences using the OneTwoTree pipeline (59), filtering intraspecific varieties, hybrids and open nomenclature. We corrected nomenclature against The Plant List (www.theplantlist.org/), which reduces the impact of poor taxonomic assignment within Genbank. OneTwoTree clustered sequences into orthology groups, which we inspected and edited by reclustering partial sequences with full sequences. Although this could have resulted in unreliable alignments, OneTwoTree selected the longest sequence for every species, and we visually inspected resulting alignments to ensure quality. We downloaded outgroup sequences from Genbank, and aligned these with ingroup sequences using Mafft -add (60). After trimming to improve quality with trimAl -gappyout command (61), we concatenated alignments into a supermatrix using AMAS (62). Finally, we removed taxa with exactly identical sequences, which are known to create short terminal branches that distort phylogenetic inference (63).

\section{Phylogenetic reconstruction}

We produced a time-calibrated phylogeny in three steps. After an initial Maximum Likelihood (ML) search using RAxML V8 (64), we identified and removed taxa exhibiting rogue behaviour in the bootstrap (BS) replicates using RogueNaRok (65). This is an especially important procedure when using the supermatrix approach as it reduces the impact of "chimeric taxa" created when sequences are misidentified and concatenated, which contain conflicting phylogenetic signal (66). We performed another ML search for the final molecular phylogeny used as input for divergence time analysis. ML searches were performed in the Cyberinfrastructure for Phylogenetic Research (67). Each ML search used 1,000 BS replicates to assess topological support, and applied an individual GTR model of nucleotide substitution to each locus partition. We enforced the monophyly of three clades in both searches: tribes Orchideae/Diseae, tribe Diurideae and tribe Cranichideae (see 11), in order to improve the likelihood calculation as in other large phylogenies (68). We calibrated the ML phylogeny against geological time using Penalized Likelihood with treePL (14). Orchids are poorly represented in the fossil record, with only one orchidoid fossil assigned with certainty, to subtribe Goodyerinae $(69,71)$. We used robust secondary calibrations (13), constraining minimum and maximum ages for 15 major clades, according to the upper and lower bounds of $95 \% \mathrm{Cls}$. An initial treePL run primed parameters for the final analysis, in which multiple smoothing parameters were tested and cross validated.

\section{Speciation analysis}

We reconstructed speciation history using the Bayesian Analysis of Macroevolutionary Mixtures (BAMM) framework (15), sampling four MCMC chains of 50 million generations every 5,000 . We set priors with the R package BAMMtools (71) and used a conservative prior of one rate shift. Rate shifts were permitted in clades of more than five species, to improve convergence. Genus-level sample fractions accounted for incomplete sampling, which were derived from a checklist (11). We excluded the first $10 \%$ of generations as burn-in, and assessed convergence with the $\mathrm{R}$ package Coda (72), by confirming the effective sample size of each parameter was $>200$. We ignored reconstructions of extinction rate, which are known to be unreliable (16). We plotted the diversification history throughout geological time with the $R$ package Strap (73).

\section{Detrended cross-correlation analyses}

We performed detrended cross-correlation analyses (DCCA) with the 9,000 post burn-in realisations of the speciation curve in $\mathrm{R}$, using code written by Davis et al (6). Briefly, we Tukeysmoothed paleoclimatic proxies, and interpolated values to the times recorded in the speciation rate curves. We calculated correlation coefficients between each of the 9,000 post burn-in speciation curves and paleoclimatic proxy, and plotted the distribution. A-1 indicates perfect negative correlation, 0 indicates no correlation, and +1 indicates perfect positive correlation (6). A Wilcoxon rank sum test assessed significance of the distribution from the null hypothesis of zero (no correlation). Note, we did not include the monotypic tribe Codonorchideae in the analysis of cross-clade consistency. Paleoclimatic proxies of global mean temperature, 
atmospheric $\mathrm{CO}_{2}$ and sea-level were sourced from Zachos et al (20), Bergmann et al (74) and Miller et al (75), respectively.

\section{Biogeographical analyses}

We downloaded georeferenced occurrences from GBIF (35) using rgbif (76), and cleaned coordinates with the $\mathrm{R}$ package CoordinateCleaner (77). We removed coordinates with uncertainty $>10 \mathrm{~km}$, and those near capital cities, biodiversity institutions, those with equal longitude and latitude values, and within seas. The resulting $>1.5$ million cleaned records were sorted into samples for analysis with Structured Rate Permutations on Phylogenies (STRAPP) (33), Es-Sim (34) and phylogenetic signal (37) calculated for binary traits with phylo.D (37) in the $\mathrm{R}$ package caper (78), and Pagel's lambda (79) for continuous traits, in the R package phytools (80). In our analysis of tropical and nontropical speciation rates, two definitions of tropical and nontropical were used, a geographical definition and a temperature definition. The geographically-defined sample defined taxa as tropical if $>50 \%$ of occurrences lie within $-/+23.5$ degrees of the equator. The temperature-defined sample assigned taxa as tropical if the yearround monthly temperature at $>50 \%$ of occurrences exceeds $18^{\circ} \mathrm{C}(81,82)$, and to calculate this we retrieved data of global temperature (1970-2000) at $1 \mathrm{~km}$ resolution, from WorldClim (83). These binary trait-dependent speciation analyses were performed with STRAPP, using 1,000 permutations and assessing significance with a Mann-Whitney test. In our analysis of speciation rates of major bioregions, we sorted taxa into seven bioregions defined by Givnish et al (36) (Africa, Australia, Europe, North America, the Pacific, and Southeast Asia), excluding taxa present in $>1$, and conducted another STRAPP test, using 1,000 permutations and assessed significance with a Kruskal-Wallis test. In our analysis of elevation-dependent speciation, we acquired minimum and maximum elevation from the Internet Orchid Species Photo Encyclopaedia (http://orchidspecies.com/: accessed 31st July 2020), and assessed elevationdependent speciation with STRAPP and Es-Sim, with both tests implementing 1,000 permutations. Es-Sim uses a Pearson test to assess significance, and we used a Spearman test in the STRAPP analysis. We tested for phylogenetic signal in these traits with Pagel's lambda, calculated using the R package phytools (80). Elevation data from orchidspecies.com is likely to be partially qualitative, but elevation data in GBIF was poor. When performing DCCA analyses for regional speciation curves, we used all 9,000 post burn-in speciation curves when investigating the role of global cooling, but used mean speciation rate when correlating regions with each other, and with atmospheric $\mathrm{CO}_{2}$ and sea-level.

\section{Transfer entropy}

Transfer Entropy is a non-parametric statistic used to infer causality between time series' (21). In the context of our analysis, TE estimates the amount of uncertainty reduced in future values of the speciation curve, by knowing past values of paleotemperature and speciation rate. We calculated TE from global mean temperature towards the mean speciation curve using the nearest-neighbour approach, implemented in the R package TransferEntropy (84). The embedding dimension was estimated using the $\mathrm{R}$ package nonlinearTseries (85). Novel code performed 1,000 permutations of the paleotemperature curve and estimated the $95 \% \mathrm{Cl}$ to test significance.

\section{Acknowledgments and funding information}

We thank the Younger laboratory (The Milner Centre for Evolution) for comments on earlier drafts. We thank the Darwin Correspondence Project. We also thank Simon Pugh Jones, MBE, DSc, president of the Orchid Society of Great Britain, for advice. This study was supported by a Roger and Sue Whorrod Studentship (to J.B.T.), John Templeton Foundation Grant 61408 (to M.A.W.) and a Research England QR GCRF grant (to N.K.P.). 


\section{References}

1. W.E. Friedman. The meaning of Darwin's 'abominable mystery'. Am. J. Bot. 96, 5-21 (2009).

2. P.S. Soltis, R.A. Folk, \& D.E. Soltis. Darwin review: angiosperm phylogeny and evolutionary radiations. Proc. R. Soc. Lond. B 286, 20190099 (2019).

3. R.J.A. Buggs. The origin of Darwin's "abominable mystery". Am. J. Bot. 108(1): 1-15 (2021).

4. O. Katz. Extending the scope of Darwin's 'abominable mystery': integrative approaches to understanding angiosperm origins and species richness. Ann. Bot. 121, 1-8 (2018).

5. R.M. Bateman. Hunting the Snark: the flawed search for mythical Jurassic angiosperms. J. Exp. Bot., 71, 22-23 (2020).

6. K.E. Davis, J. Hill, T.I. Astrop \& M.A. Wills. Global cooling as a driver of diversification in a major marine clade. Nat. Commun., 7, 1-8. (2016).

7. M. Sun et al. Recent accelerated diversification in rosids occurred outside the tropics. Nat. Commun. 11, 3333 (2020).

8. C. Tang, K.E. Davis, C. Delmer, D. Yang \& M.A. Wills. Elevated atmospheric $\mathrm{CO}_{2}$ promoted speciation in mosquitoes (Diptera, Culicidae). Commun. Biol. 1, 1-8 (2018).

9. M. Cardillo \& R. Pratt. Evolution of a hotspot genus: geographic variation in speciation and extinction rates in Banksia (Proteaceae). BMC Evol. Biol. 13, 155 (2013).

10. Y.Y. Guo, Y.B. Luo, Z.J. Liu \& X.Q. Wang. Reticulate evolution and sea-level fluctuations together drove species diversification of slipper orchids (Paphiopedilum) in South-East Asia. Mol. Ecol. 24, 2838-2855 (2015).

11. M.W. Chase et al. An updated classification of Orchidaceae. Bot. J. Linn. Soc. 177, 151-174 (2015).

12. C. Darwin. On the various contrivances by which British and foreign orchids are fertilised by insects. John Murray (1862).

13. T.J. Givnish et al. Orchid phylogenomics and multiple drivers of their extraordinary diversification. Proc. R. Soc. B. 282, 1553 (2015).

14. S.A. Smith \& B.C. O'Meara. treePL: divergence time estimation using penalized likelihood for large phylogenies. Bioinform. 28, 2689-2690 (2012).

15. D.L. Rabosky. Automatic detection of key innovations, rate shifts, and diversity- dependence on phylogenetic trees. PLOS ONE 9: e89543 (2014).

16. D.L. Rabosky. Extinction rates should not be estimated from molecular phylogenies. Evol. 64, 1816-1824 (2010).

17. D.L. Rabosky. Challenges in the estimation of extinction from molecular phylogenies: a response to Beaulieu and O'Meara. Evol. 70, 218-228 (2016).

18. B.R. Moore, S. Höhna, M.R. May, B. Rannala and J.P. Huelsenbeck. Critically evaluating the theory and performance of Bayesian analysis of macroevolutionary mixtures. Proc. Natl. Acad. Sci. U.S.A. 113, 9569-9574 (2016).

19. D.L. Rabosky, J.S. Mitchell \& J. Chang. Is BAMM flawed? Theoretical and practical concerns in the analysis of multi-rate diversification models. Syst. Biol., 66, 477-498 (2017).

20. J. Zachos, M. Pagani, L. Sloan, E. Thomas \& K. Billups. Trends, rhythms, and aberrations in global climate 65 Ma to present. Science, 292, 686-693 (2001).

21. T. Schreiber. Measuring information transfer. Phys. Rev. Lett. 85, 461 (2000). 
22. K.E. Davis, A.T. Bakewell, J. Hill, H. Song \& P. Mayhew. Global cooling \& the rise of modern grasslands: Revealing cause \& effect of environmental change on insect diversification dynamics. BioRxiv. 392712 (2018).

23. M. Chávez, J. Martinerie \& M. Le Van Quyen. Statistical assessment of nonlinear causality: application to epileptic EEG signals. J. Neurosci. Methods. 124, 113-128 (2003).

24. G.L. Foster \& E.J. Rohling. Relationship between sea level and climate forcing by $\mathrm{CO}_{2}$ on geological timescales. Proc. Natl. Acad. Sci. U.S.A. 110, 1209-1214 (2013).

25. X. Wang et al. Genetic, phenotypic and ecological differentiation suggests incipient speciation in two Charadrius plovers along the Chinese coast. BMC Evol. Biol. 19, 135 (2019).

26. J.J. Shelley et al. Plio-Pleistocene sea-level changes drive speciation of freshwater fishes in north-western Australia. J. Biogeogr. 47, 1727-1738 (2020).

27. E. Newman, B. Anderson, \& S.D. Johnson. Flower colour adaptation in a mimetic orchid. Proc. Biol. Sci. B 279, 2309-13 (2012).

28. S.D. Johnson, N. Hobbhahn \& B. Bytebier. Ancestral deceit and labile evolution of nectar production in the African orchid genus Disa. Biol. Lett. 9, 20130500 (2013).

29. J. Igea \& A.J. Tanentzap. Angiosperm speciation cools down in the tropics. Ecol. Lett. 23, 692-700 (2020).

30. C. Hughes \& R. Eastwood. Island radiation on a continental scale: exceptional rates of plant diversification after uplift of the Andes. Proc. Natl. Acad. Sci. U.S.A. 103, 10334-10339 (2006).

31. C. Hoorn et al. Amazonia through time: Andean uplift, climate change, landscape evolution, and biodiversity. Science 330, 927-931 (2010).

32. O.A. Pérez-Escobar et al. Recent origin and rapid speciation of Neotropical orchids in the world's richest plant biodiversity hotspot. New Phytol. 215, 891-905 (2017).

33. D.L. Rabosky \& H. Huang. A robust semi-parametric test for detecting trait-dependent diversification. Syst. Biol., 65, 181-193 (2016).

34. M.G. Harvey \& D.L. Rabosky. Continuous traits and speciation rates: alternatives to statedependent diversification models. Methods Ecol. Evol. 9, 984-993 (2018).

35. GBIF.org. GBIF Occurrence Download https://doi.org/10.15468/dl.q6jft4. (03 August 2020).

36. T.J. Givnish et al. Orchid historical biogeography, diversification, Antarctica and the paradox of orchid dispersal. J. Biogeogr. 43, 1905-1916 (2016).

37. S.A. Fritz \& A. Purvis. Selectivity in mammalian extinction risk and threat types: a new measure of phylogenetic signal strength in binary traits. Cons. Biol. 24, 1042-1051 (2010).

38. T.J. Davies et al. Darwin's abominable mystery: Insights from a supertree of the angiosperms. Proc. Natl. Acad. Sci. U.S.A. 101, 1904-1909 (2004).

39. S.J. Ivory et al. Environmental change explains cichlid adaptive radiation at Lake Malawi over the past 1.2 million years. Proc. Natl. Acad. Sci. U.S.A. 113, 11895-11900 (2016).

40. M.D. McGee et al. The ecological and genomic basis of explosive adaptive radiation. Nature 586, 75-79 (2020).

41. B.R. Grant \& P.R. Grant. Evolution of Darwin's finches caused by a rare climatic event. Proc. R. Soc. Lond. B. 251, 111-117 (1993).

42. S.C. Campbell-Staton et al. Winter storms drive rapid phenotypic, regulatory, and genomic shifts in the green anole lizard. Science. 357, 495-498 (2017). 
43. J.A. Chaves et al. Genomic variation at the tips of the adaptive radiation of Darwin's finches. Mol. Ecol. 25, 5282-5295 (2016).

44. Y.E. Stuart et al. Rapid evolution of a native species following invasion by a congener. Science 346, 463-466 (2014).

45. A.C. Chaboureau et al. Tectonic-driven climate change and the diversification of angiosperms. Proc. Natl. Acad. Sci. USA. 111, 14066-14070 (2014).

46. T. Hernández-Hernández \& J.J. Wiens. Why are there so many flowering plants? A multiscale analysis of plant diversification. Am. Nat. 195, 948-963 (2020).

47. C.P. Osborne. Atmosphere, ecology and evolution: what drove the Miocene expansion of C4 grasslands? J. Ecol. 96, 35-45 (2008).

48. J.W. Andrae et al. Initial expansion of $\mathrm{C} 4$ vegetation in Australia during the late Pliocene. Geophys. Res. Lett., 45, 4831-4840 (2018).

49. W. Zhong \& N.K. Priest. Stress-induced recombination and the mechanism of evolvability. Behav. Ecol. and Sociobiol. 65, 493-502 (2011).

50. A. Lloyd, C. Morgan., F.C.H. Franklin \& K. Bomblies, Plasticity of Meiotic Recombination Rates in Response to Temperature in Arabidopsis. Genetics 208, 1409-1420 (2018).

51. P. Šmarda et al. Ecological and evolutionary significance of genomic GC content diversity in monocots. Proc. Natl. Acad. Sci. U.S.A. 111, E4096-E4102 (2014).

52. P. Trávníček et al. Diversity in genome size and GC content shows adaptive potential in orchids and is closely linked to partial endoreplication, plant life-history traits and climatic conditions. New Phytol. 224, 1642-1656 (2019).

53. F. Balao et al. Adaptive sequence evolution is driven by biotic stress in a pair of orchid species (Dactylorhiza) with distinct ecological optima. Mol. Ecol. 26, 3649-3662 (2017).

54. M.K. Brandrud et al. Phylogenomic Relationships of Diploids and the Origins of Allotetraploids in Dactylorhiza (Orchidaceae). Syst. Biol. 69, 91-109 (2020).

55. J.R. Etterson \& R.G. Shaw. Constraint to adaptive evolution in response to global warming. Science. 294, 151-154 (2001).

56. C. Hof, I. Levinsky, M.B. Araújo \& C. Rahbek. Rethinking species' ability to cope with rapid climate change. Glob. Chang. Biol. 17, 2987- 2990 (2011).

57. N. Jones, B. Husband, \& A. MacDougall. Reproductive system of a mixed-mating plant responds to climate perturbation by increased selfing. Proc. Royal Soc. B 280, 20131336 (2013).

58. W.K. Petry et al. Sex-specific responses to climate change in plants alter population sex ratio and performance. Science 353, 69-71 (2016).

59. M. Drori et al. OneTwoTree: An online tool for phylogeny reconstruction. Mol. Ecol. Resour. 18, 1492-1499 (2018).

60. K. Katoh \& M.C. Frith. Adding unaligned sequences into an existing alignment using MAFFT and LAST. Bioinformatics 28, 3144-3146 (2012).

61. S. Capella-Gutierrez, J.M. Silla-Martinez, T. Gabaldon. trimAl: a tool for automated alignment trimming in large-scale phylogenetic analyses. Bioinformatics. 25, 1972-1973 (2009).

62. L.M. Borowiec. AMAS: a fast tool for alignment manipulation and computing of summary statistics. PeerJ. 4, e1660 (2016).

63. N.M. Reid \& B.C. Carstens. Phylogenetic estimation error can decrease the accuracy of species delimitation: a Bayesian implementation of the general mixed Yule-coalescent model. BMC Evol. Biol. 12, 196 (2012). 
64. A. Stamatakis. RAxML version 8: a tool for phylogenetic analysis and post-analysis of large phylogenies. Bioinformatics, 30, 1312-1313 (2014).

65. A.J. Aberer, D. Krompass \& A. Stamatakis. Pruning rogue taxa improves phylogenetic accuracy: an efficient algorithm and webservice. Syst. Biol. 62, 162-166 (2013).

66. C.E. Hinchliff \& E.H. Roalson. Using supermatrices for phylogenetic inquiry: an example using the sedges. Syst. Biol., 62, 205-219 (2013).

67. M.A. Miller, W. Pfeiffer \& T. Schwartz. Creating the CIPRES science gateway for inference of large phylogenetic trees. In Gateway Computing Environments Workshop (GCE), New Orleans, LA, 1-8 (2010).

68. J.J. Shi \& D.L. Rabosky. Speciation dynamics during the global radiation of extant bats. Evolution. 69, pp.1528-1545 (2015).

69. S.R. Ramírez, B. Gravendeel, R.B. Singer, C.R. Marshall \& N.E. Pierce. Dating the origin of the Orchidaceae from a fossil orchid with its pollinator. Nature, 448, 1042-1045 (2007).

70. A.L.S. Gustafsson, C.F. Verola \& A. Antonelli. Reassessing the temporal evolution of orchids with new fossils and a Bayesian relaxed clock, with implications for the diversification of the rare South American genus Hoffmannseggella (Orchidaceae: Epidendroideae). BMC Evol. Biol. 10, 177 (2010).

71. D.L. Rabosky et al. BAMM tools: an R package for the analysis of evolutionary dynamics on phylogenetic trees. Methods Ecol. Evol. 5, 701-707 (2014).

72. M. Plummer, N. Best, K. Cowles \& K. Vines. CODA: Convergence Diagnosis and Output Analysis for MCMC. R. News 6, 7-11 (2006).

73. M.A. Bell \& G.T. Lloyd. strap: an R package for plotting phylogenies against stratigraphy and assessing their stratigraphic congruence. Palaeontology. 58, 379-389 (2015).

74. N.M. Bergman, T.M. Lenton \& A.L. Watson. Copse: A new model of biogeochemical cycling over Phanerozoic time. Am. J. Sci. 304, 397-437 (2004).

75. K.G. Miller et al. Cenozoic sea-level and cryospheric evolution from deep-sea geochemical and continental margin records. Sci. Adv. 6, p.eaaz1346 (2020).

76. S. Chamberlain. et al. rgbif: Interface to the Global Biodiversity Information Facility API. R package version 1.3.0. https://CRAN.R-project.org/package=rgbif (2019).

77. A. Zizka et al. CoordinateCleaner: automated cleaning of occurrence records from biological collections. $\mathrm{R}$ package v1.0.7. https://CRAN.R-project.org/package=CoordinateCleaner (2018).

78. D. Orme et al. The caper package: comparative analysis of phylogenetics and evolution in R. $R$ package version 5, 1-36 (2013).

79. M. Pagel. Inferring the historical patterns of biological evolution. Nature 401, 877-884 (1999).

80. L.J. Revell. phytools: an R package for phylogenetic comparative biology (and other things). Methods Ecol. Evol. 3, 217-223 (2011).

81. H.L. Owens et al. The latitudinal diversity gradient in New World swallowtail butterflies is caused by contrasting patterns of out-of-and into-the-tropics dispersal. Glob. Ecol. Biogeogr. 26, 1447-1458 (2017).

82. M.C. Peel, B.L. Finlayson. \& T.A. McMahon. Updated world map of the Köppen-Geiger climate classification. Hydrol. Earth Syst. Sci. 11, 1633-1644 (2007).

83. S.E. Fick \& R.J. Hijmans. WorldClim 2: new 1-km spatial resolution climate surfaces for global land areas. Int. J. Climatol. 37, 4302-4315 (2017).

84. G.H. Torbati \& G. Lawyer. TransferEntropy: The Transfer Entropy Package, https://CRAN.Rproject.org/package=TransferEntropy (2016). 
bioRxiv preprint doi: https://doi.org/10.1101/2021.02.06.430029; this version posted February 8, 2021. The copyright holder for this preprint (which was not certified by peer review) is the author/funder. All rights reserved. No reuse allowed without permission.

85. C.A. Garcia \& G. Sawitzki. nonlinearTseries: Nonlinear Time Series Analysis, https://CRAN.R-project.org/package=nonlinearTseries (2015). 
Figures and Tables

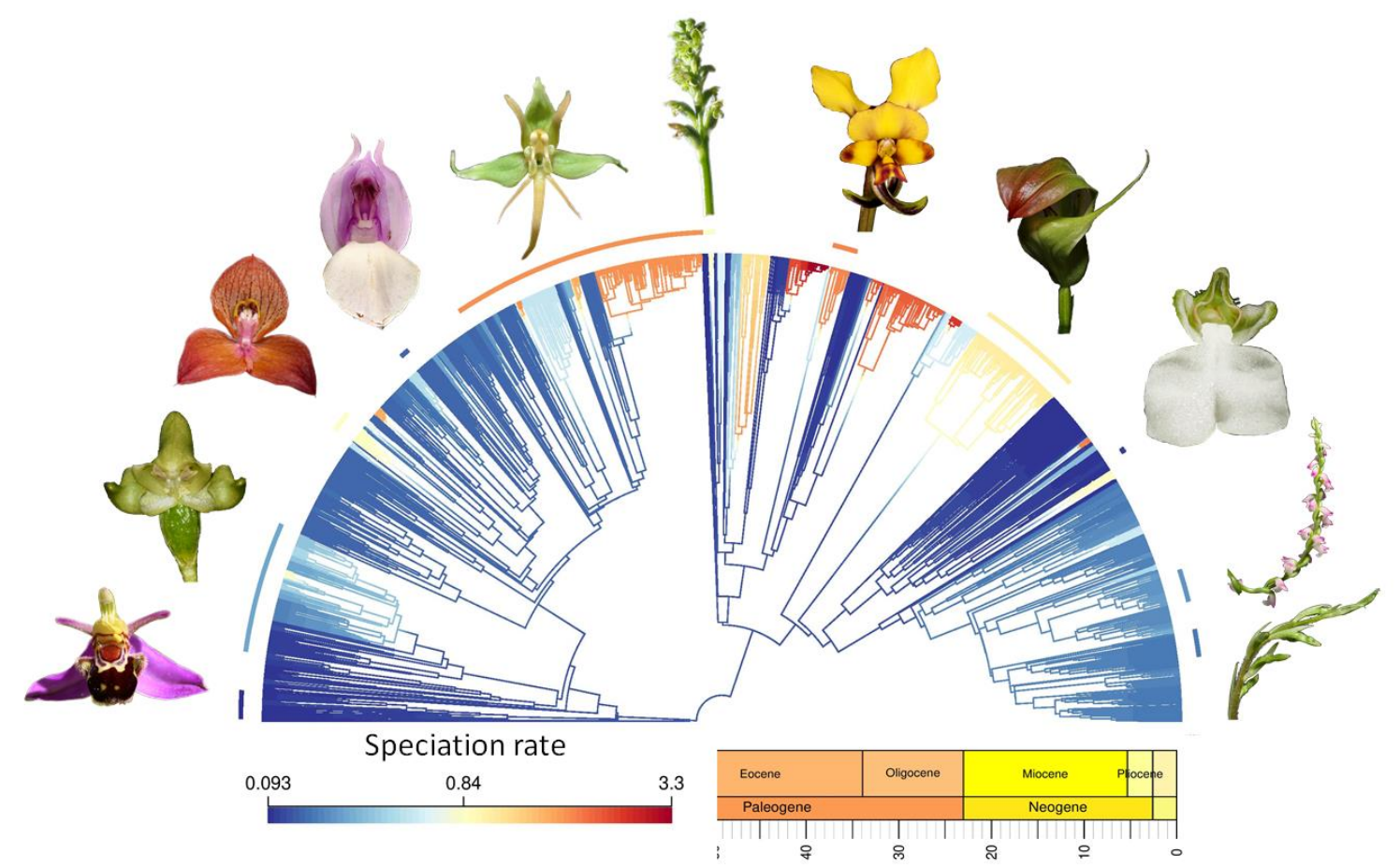

Figure 1: Time-calibrated phylogeny of 1,450 orchidoid taxa, visualised against the geological timescale. Branches are coloured by speciation rates estimated with Bayesian Analysis of Macroevolutionary Mixtures. Iconic orchidoid genera representing the diversity of form are associated with arc segments coloured by mean speciation rate. Images were sourced from Flickr (Creative Commons and modifications allowed). 

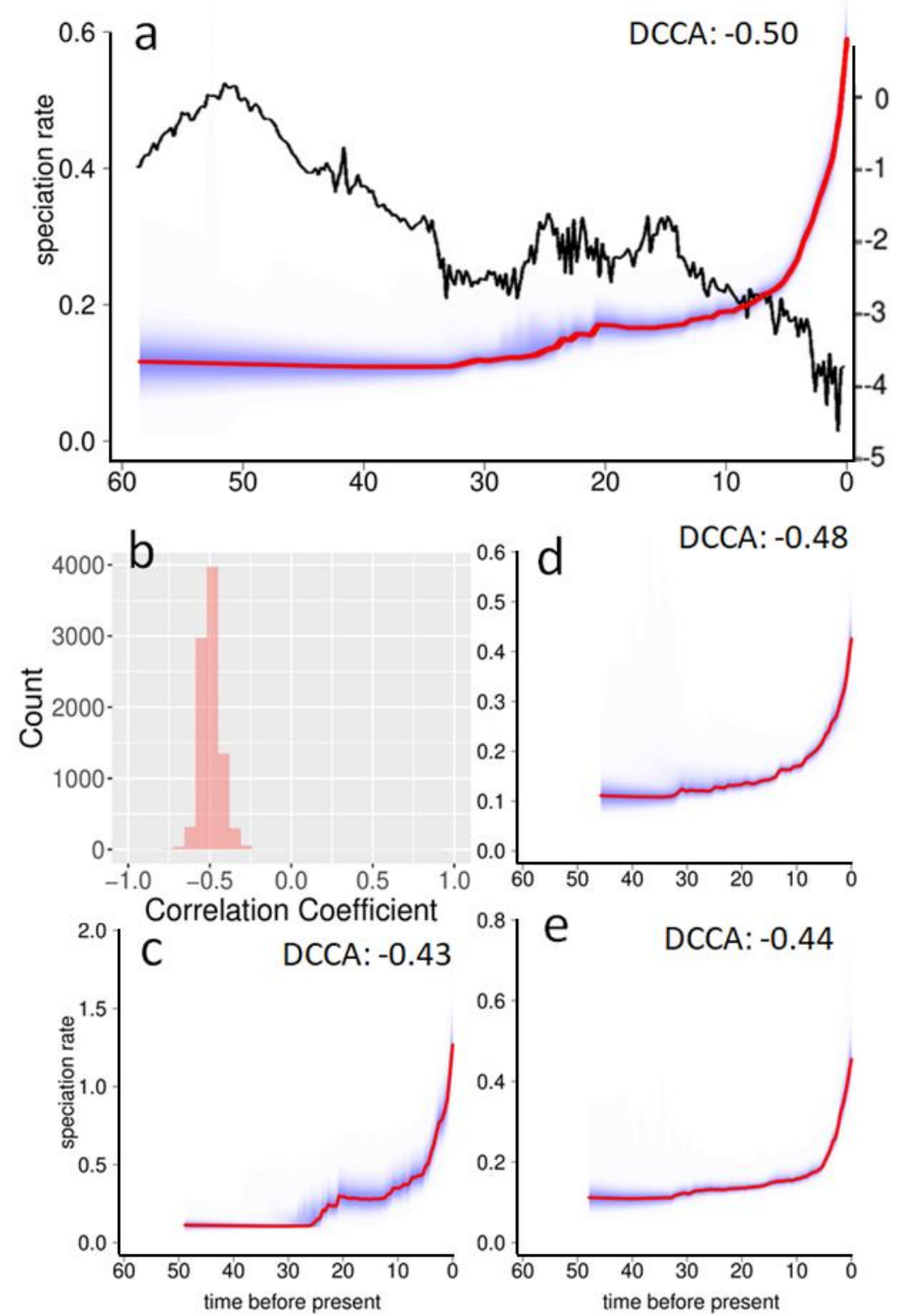

Figure 2. Consistency in the influence of global cooling on rapid diversification across Orchidoideae. The estimated historical speciation rate curve for the whole tree (red line with blue $95 \% \mathrm{Cl})$ is plotted alongside the curve of estimated mean global paleotemperature $\left(\delta^{18} \mathrm{O}\right.$, black line) (a). A histogram of the DCCA coefficients used to infer the mean correlation. coefficient across the whole tree (b). The estimated historical speciation rate curves of sub-clades Diurideae (c), Cranichideae (d) and Orchideae/Diseae (e) are reported with mean correlation coefficients indicated (all are consistently negative and significant at $\mathrm{p}<0.0001$ ). 

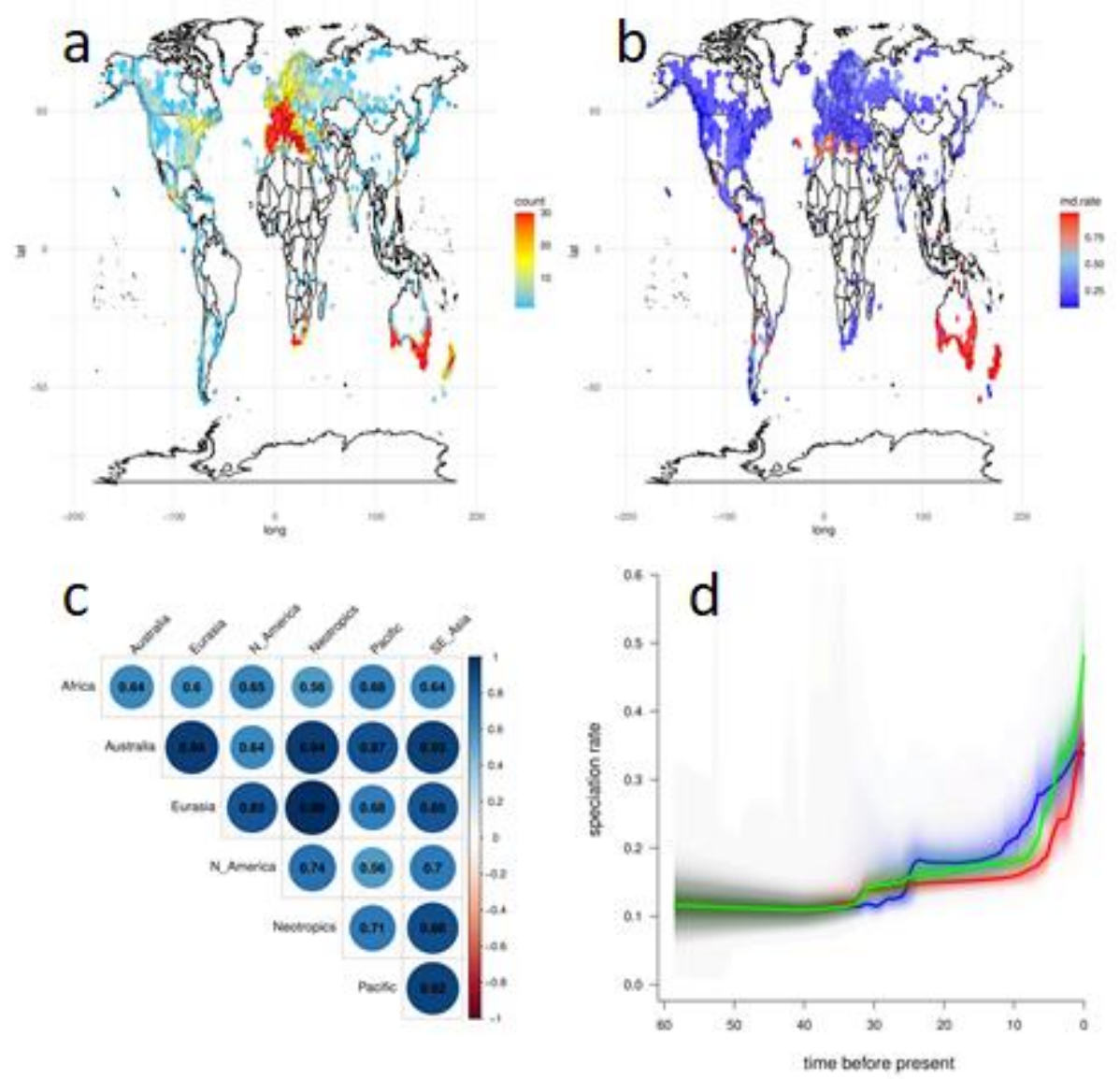

Figure 3: Geographic consistency in diversification driven by global cooling. Global maps of species richness (a) and speciation rate (b) are plotted to a standard 200 square mile grid with levels indicated by colour keys. The correlation matrix of speciation rate through time curves of endemic subclades from each of the major orchid bioregions (correlation range from light blue to dark blue, +0.56 to +0.99 , respectively) reveals that diversification happened independently and simultaneously across the earth (c). An example of rate-through-time curves of endemic lineages from the "Eurasia" (red), "Neotropics" (green), and "Southeast Asia" (blue) major orchid bioregions (d). 
Table 1. The consequences of atmospheric $\mathrm{CO}_{2}$ and sea-level vary by geography. DCCA correlations between speciation rate and climate indices are reported with significance indicated $\left({ }^{*}=p<0.05,{ }^{* *}=p<0.001\right)$.

\begin{tabular}{|l|l|l|}
\hline Bioregion & Atmospheric $\mathrm{CO}_{2}$ & Sea-level \\
\hline Africa & -0.18 & $0.36^{* *}$ \\
\hline Australia & 0.078 & 0.046 \\
\hline Eurasia & -0.16 & 0.14 \\
\hline Neotropics & -0.098 & 0.078 \\
\hline North America & -0.27 & $0.57^{\star *}$ \\
\hline Pacific & $0.37^{* *}$ & 0.074 \\
\hline Southeast Asia & $0.20^{*}$ & 0.074 \\
\hline
\end{tabular}

